\title{
A bench study comparing between scalpel-bougie technique and cannula-to-Melker technique in emergency cricothyroidotomy in a porcine model
}

\author{
See Seong Chang ${ }^{1}$, Qian Jun Tong ${ }^{1}$, Zhi Yuen Beh ${ }^{2}$, Kelvin Howyow Quek ${ }^{1}$, \\ and Bun Hui Ang ${ }^{1}$ \\ ${ }^{1}$ Department of Anaesthesia and Surgical Intensive Care, Changi General Hospital, Singapore, ${ }^{2}$ Department of \\ Anaesthesia, University of Malaya Faculty of Medicine, Kuala Lumpur, Malaysia
}

\begin{abstract}
Background: The ideal emergency cricothyroidotomy technique remains a topic of ongoing debate. This study aimed to compare the cannula-to-Melker technique with the scalpel-bougie technique and determine whether yearly training in cricothyroidotomy techniques is sufficient for skill retention.

Methods: We conducted an observational crossover bench study to compare the cannula-to-Melker with the scalpel-bougie technique in a porcine tracheal model. Twenty-eight anesthetists participated. The primary outcome was time taken for device insertion. Secondary outcomes were first-pass success rate, incidence of tracheal trauma, and technique preference. We also compared the data on outcome measures with the data obtained in a similar workshop a year ago. Results: The scalpel-bougie technique was significantly faster than the cannula-to-Melker technique for cricothyroidotomy (median time of $45.2 \mathrm{~s}$ vs. $101.3 \mathrm{~s} ; \mathrm{P}=0.001$ ). Both techniques had $100 \%$ success rate within two attempts; there were no significant differences in the first-pass success rates and incidence of tracheal wall trauma $(\mathrm{P}>0.999$ and $\mathrm{P}=0.727$, respectively) between them. The relative risks of inflicting tracheal wall trauma after a failed cricothyroidotomy attempt were 6.9 (95\% CI 1.5-31.1), 2.3 (95\% CI 0.3-20.7) and 3.0 (95\% CI 0.3-25.9) for the scalpel-bougie, cannula-cricothyroidotomy, and Melker-Seldinger airway, respectively. The insertion time and incidence of tracheal wall trauma were lower when the present data were compared with data from a similar workshop conducted the previous year.

Conclusions: This study supports the use of a scalpel-bougie technique for cricothyroidotomy by anesthetists and advocates a yearly training program for skill retention.
\end{abstract}

Keywords: Airway management; Intubation; Trachea; Tracheostomy.

Corresponding author: See Seong Chang, BMed Sc, MBBS, MMed (Anaes)

Department of Anaesthesia and Surgical Intensive Care, Changi General Hospital, 2 Simei Street 3, 529889, Singapore

Tel: 6568501951, Fax: 6562601693, Email: chang.see.seong@singhealth.com.sg

ORCID: https://orcid.org/0000-0002-3240-3707

Received: January 30, 2018. Revised: May 7, 2018. Accepted: May 21, 2018.

Korean J Anesthesiol 2018 August 71(4): 289-295

https://doi.org/10.4097/kja.d.18.00025

(c) This is an open-access article distributed under the terms of the Creative Commons Attribution Non-Commercial License (http://creativecommons.org/ licenses/by-nc/4.0/), which permits unrestricted non-commercial use, distribution, and reproduction in any medium, provided the original work is properly cited. 


\section{Introduction}

A "cannot intubate, cannot oxygenate" (CICO) event occurs when the traditional methods of maintaining airway patency and oxygenation fail. The event can result in serious morbidity and mortality if not properly managed [1]. However, due to its rarity $(1: 50,000)$ [2], most anesthetists have little experience and are not confident with cricothyroidotomy techniques to maintain oxygenation [3]. Regular training is, therefore, recommended to reinforce and retain skills [1].

The ideal cricothyroidotomy technique should be easy and quick to perform, have high success rates and low complication rates, and allow adequate ventilation [4]. Two common cricothyroidotomy techniques are the cannula-to-Melker (CM) technique and the scalpel-bougie (SB) technique. Although the recent 2015 Difficult Airway Society (DAS) Guidelines [1] recommend the SB as the default technique for cricothyroidotomy, the most appropriate technique remains a topic of debate [5]. Advantages of the CM technique include its ease of use and the fact that anesthetists are more familiar with handling cannulas than scalpels [1]. The disadvantages of the CM technique include its failure because of malposition, displacement, or kinking of the cannula [6]. On the other hand, the SB technique has been shown to have a high success rate [7]; however, there are fears that anesthetists may be reluctant to use a scalpel, and this will lead to delayed interventions [6].

The primary aim of this study was to compare the CM and the SB techniques using a porcine tracheal model. The secondary aim was to determine if yearly training in cricothyroidotomy techniques is sufficient to reinforce and retain skills.

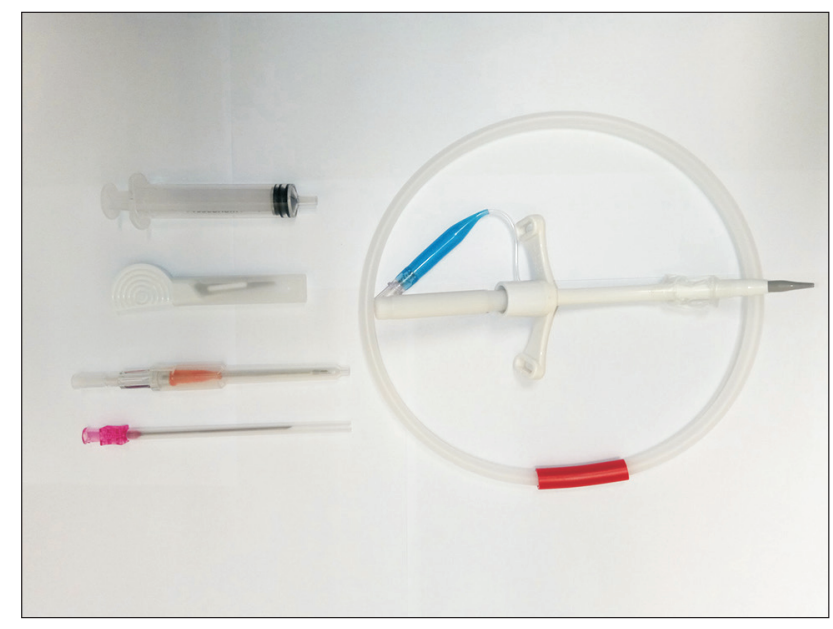

Fig. 1. Equipment for the cannula-to-Melker technique.

\section{Materials and Methods}

An ethics boards review exemption was granted by the SingHealth Centralized Institutional Review Board D (CIRB Ref: 2017/2878). The requirement for written informed consent was waived.

We conducted an observational crossover bench study to compare between the CM technique and the SB technique in a porcine tracheal model. This study was reported in accordance to the Transparent Reporting of Evaluations with Nonrandomized Designs statement [8].

The participants were recruited from a one-day departmental CICO workshop that was held on October 28, 2017. The participants of the workshop were mostly consultant anesthetists, with a few senior anesthesia trainees from the Department of Anesthesia, Changi General Hospital, Singapore. All participants of the study first completed a questionnaire to determine their years of practice in anesthesia, their most recent training experience in cricothyroidotomy techniques, and whether they participated in a similar departmental CICO workshop conducted the previous year.

Reading materials and online video links were provided to all participants one week before the workshop. At the workshop, each cricothyroidotomy technique was demonstrated live in a stepwise manner by the faculty members. The participants were then asked to perform emergency cricothyroidotomy using both techniques in turn on a fresh porcine trachea each time.

For the CM technique, we used a 14-gauge BD Insyte ${ }^{\mathrm{TM}}$ intravenous (IV) cannula (Becton, Dickinson and Company, USA) and the Melker emergency cricothyroidotomy catheter set (Cook Medical LLC, USA) with a $5.0 \mathrm{~mm}$ cuffed tube (Fig. 1). For the SB technique, we used a size 10 scalpel, a Portex 15Fr coude-tipped bougie (Smiths Medical, USA) and a $6.0 \mathrm{~mm}$

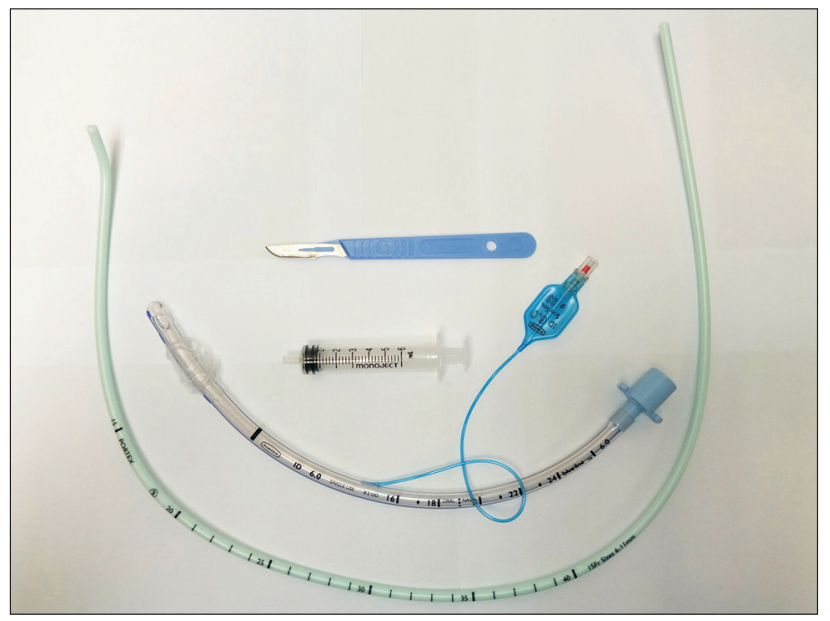

Fig. 2. Equipment for scalpel-bougie technique. 
Portex cuffed endotracheal tube (Smiths Medical, USA) (Fig. 2 ). The porcine model comprised of a pig larynx with a trachea that was secured by the workshop faculty members. This model allowed for palpation of the underlying laryngeal anatomy.

The CM technique consisted of two parts: the initial cricothyroid puncture, followed by the Melker Seldinger technique as described by Heard et al. [7]. For the SB technique, we employed the simple scalpel "stab, twist, bougie, tube" technique with a "laryngeal handshake" to locate the cricothyroid membrane as advocated in the 2015 DAS Guidelines [1].

The primary outcome was the time taken for successful placement of an airway device in the porcine trachea. For the CM technique, two sets of timings were recorded by trained, independent observers. The first set was taken for the cannula cricothyroidotomy. The timer was started when the participants were instructed to perform the technique, and the timer was stopped when the cricothyroid puncture was successful as indicated by free aspiration of air from the IV cannula. The second set of timings was recorded for insertion of the Melker Seldinger airway. The timer was started when the participant picked up the guidewire and stopped when the $5.0 \mathrm{~mm}$ tube cuff was inflated. For the SB technique, the timer was started when the participants were instructed to perform the technique and stopped when the cuff of the $6.0 \mathrm{~mm}$ endotracheal tube was inflated. The SB technique was deemed a failure when the time reached $300 \mathrm{~s}$. The CM technique was deemed a failure when the combined time for the cannula cricothyroidotomy and the Melker Seldinger airway reached $300 \mathrm{~s}$. There was no limit to the total number of attempts.

The secondary outcomes were the first-pass success rate and the incidence of posterior tracheal wall trauma. The presence of posterior tracheal wall trauma was determined with the airway device removed and the trachea dissected for inspection. The data of the primary and secondary outcomes were also compared with the recorded data obtained from a similar departmental CICO workshop conducted the previous year.

At the conclusion of the study, the participants were requested to fill a post-study questionnaire to indicate their preferred technique and confidence in performing these techniques using a numeric rating scale $(0=$ minimum to $10=$ maximum $)$.

\section{Statistical analysis}

Descriptive statistics were used to describe the primary and secondary outcomes followed by appropriate statistical testing. Frequencies with percentages, means and standard deviations, or medians and interquartile ranges (IQRs)/ranges were reported as appropriate. The McNemar's test was used to compare paired categorical data. The Fisher's exact test was used to assess the associations between two independent categorical data. The
Wilcoxon signed-rank test was used to compare the insertion times and the pre-post confidence level for the two cricothyroidotomy techniques. The Mann-Whitney $U$ test was used to compare numeric data between two independent groups.

A two tailed, $\mathrm{P}$ value of $<0.05$ was considered statistically significant. Statistical analysis was performed using IBM SPSS Statistics, version 19 (International Business Machines Corp., USA).

\section{Results}

\section{Participant characteristics}

Thirty anesthetists took part in the departmental CICO workshop. Twenty-eight anesthetists consented to participate in the study. Of these participants, 21 were consultant anesthetists, 2 were fellows, 2 were final year anesthesia trainees, and 3 were trainee anesthetists. Twenty-three participants (82.1\%) attended the departmental CICO workshop in the previous year. Table 1 illustrates the participant characteristics and experience.

\section{Primary outcome}

There were no failed procedures for both SB and CM techniques (defined as procedure time $>300 \mathrm{~s}$ ). The $\mathrm{SB}$ technique was significantly quicker to perform than the $\mathrm{CM}$ technique $(\mathrm{P}=0.001$, when time to tube placement of the SB technique was compared to the total time required for the cannula cricothyroidotomy and the Melker Seldinger airway). Details of the duration of insertion for both techniques are shown in Table 2 .

Table 1. Participant Characteristics and Experience

\begin{tabular}{lc}
\multicolumn{1}{c}{ Anesthetist and experience } & $\mathrm{n}=28$ \\
\hline Years in anesthesia & $1(3.6)$ \\
$0-5$ & $16(57.1)$ \\
$6-10$ & $6(21.4)$ \\
$11-15$ & $5(17.9)$ \\
More than 15 & \\
Prior training to perform scalpel-bougie (if any) & $13(46.4)$ \\
Within 12 months & $10(35.7)$ \\
$1-5$ years & $3(10.7)$ \\
More than 5 years & $2(7.1)$ \\
Never & $11(39.3)$ \\
Prior training to perform cannula-Melker (if any) & $10(35.7)$ \\
Within 12 months & $1(3.6)$ \\
1-5 years & $6(21.4)$ \\
More than 5 years & \\
Never &
\end{tabular}

Values are expressed as number (\%). 


\section{Secondary outcome}

There were no significant statistical differences in the first pass success rates and in the incidence of posterior tracheal wall trauma between the two techniques. The details are shown in Table 2.

For the SB technique, participants who required more than one attempt had about seven (relative risk 6.9; 95\% CI 1.5-31.1) times the risk of causing a posterior tracheal wall trauma compared to those who required only one attempt $(3 / 5,60.0 \%$ vs. $2 / 23,8.7 \%$; $[\mathrm{P}=0.027]$ ) (median number of attempts $=1.0$; range 1-4).

For the cannula cricothyroidotomy technique, the relative risk of posterior tracheal wall trauma if the participant required more than one attempt was $2.3(1 / 5,20.0 \%$ vs. $2 / 23,8.7 \%$; $95 \%$ CI $0.3-20.7 ; \mathrm{P}=0.459$ ) (median number of attempts $=1.0$; range $1-3)$.

For the Melker Seldinger airway, the relative risk of posterior tracheal wall trauma if the participant required more than one attempt was $3.0(1 / 4,25.0 \%$ vs. $2 / 24,8.3 \%$; $95 \%$ CI $0.3-25.9 ; \mathrm{P}=$ 0.382 ) (median number of attempts $=1.0$; range $1-3$ ).

Table 3 shows the primary and secondary outcome data when compared with the recorded data obtained from a similar departmental CICO workshop conducted the previous year. Although statistically insignificant, the results suggested a trend towards shorter duration of insertion when the time to tube placement for both SB and CM techniques from the 2017 CICO workshop was compared with data from the 2016 workshop. There was also a trend towards a lower incidence of posterior tracheal wall trauma for both techniques in the most recent CICO workshop.

The post-study questionnaire showed that SB technique was the preferred method of cricothyroidotomy as shown in Table 4. There was statistically significant improvement in the self-rated confidence in performing both cricothyroidotomy techniques ( $\mathrm{P}$ $<0.001)$. The results are illustrated in Table 5.

\section{Discussion}

In this study, the median insertion time for the SB technique (45.2 s) was found to be significantly $(P=0.001)$ shorter than that for the CM technique (101.3 s). This is consistent with the findings reported in a previous meta-analysis [9]. The SB technique was also the preferred method of cricothyroidotomy for most participants (64\%).

All participants successfully performed either cricothyroidot-

Table 2. Primary and Secondary Outcomes

\begin{tabular}{lcccrr}
\hline \multirow{2}{*}{ Outcomes } & & \multicolumn{3}{c}{ Cannula-to-Melker conversion } & P value \\
\cline { 3 - 5 } & Scalpel-bougie & Cannula & Melker seldinger & Total & \\
\hline Duration of insertion $(\mathrm{s})$ & $45(38-76)$ & $33(22-44)$ & $57(52-76)$ & $101(81-115)$ & $0.001^{*}$ \\
First-pass success & $23(82.1)$ & $23(82.1)$ & $24(85.7)$ & $\mathrm{n}^{\mathrm{a}}$ & $>0.999^{\dagger}$ \\
Presence of posterior wall trauma & $5(17.9)$ & & $3(10.7)$ & $0.727^{\dagger}$ \\
\hline
\end{tabular}

Values are expressed as median (IQR) or number (\%). *Wilcoxon signed-rank test was used to compare the duration of scalpel-bougie and total duration for cannula-to-Melker conversion; ${ }^{\dagger} \mathrm{McNemar}$ 's test was used to compare the scalpel-bougie and cannula-to-Melker conversion. n/a: not applicable.

Table 3. Comparison of Primary and Secondary Outcomes with Data from a Previous CICO Workshop

\begin{tabular}{|c|c|c|c|c|c|}
\hline \multirow{2}{*}{ Outcomes } & \multirow{2}{*}{ Year } & \multirow{2}{*}{ SB } & \multicolumn{3}{|c|}{ Cannula-to-Melker conversion } \\
\hline & & & Cannula & Melker seldinger & Total \\
\hline \multirow[t]{3}{*}{ Time to tube placement (s) } & 2016 & $49(35-77)$ & $37(21-54)$ & $65(46-78)$ & $113(81-138)$ \\
\hline & 2017 & $45(38-76)$ & $33(22-44)$ & $57(52-76)$ & $101(81-115)$ \\
\hline & $P$ value & 0.952 & 0.531 & 0.888 & 0.342 \\
\hline \multirow[t]{3}{*}{ First-pass success } & 2016 & $18(85.7)$ & $\mathrm{n} / \mathrm{a}$ & $\mathrm{n} / \mathrm{a}$ & $13(61.9)$ \\
\hline & 2017 & $23(82.1)$ & $23(82.1)$ & $24(85.7)$ & $\mathrm{n} / \mathrm{a}$ \\
\hline & $P$ value & $>0.999$ & & & \\
\hline \multirow[t]{3}{*}{ Presence of partial posterior wall trauma } & 2016 & $7(33.3)$ & & $5(23.8)$ & \\
\hline & 2017 & $5(17.9)$ & & $3(10.7)$ & \\
\hline & $P$ value & 0.213 & & 0.263 & \\
\hline
\end{tabular}

Values are expressed as median (IQR) or number (\%). CICO: cannot intubate, cannot oxygenate, SB: scalpel-bougie, CM: cannula-to-Melker, n/a: not applicable. 
Table 4. Infraglottic Rescue Technique Preference

\begin{tabular}{lccc}
\hline \multicolumn{1}{c}{ Parameters } & SB & CM & P value \\
\hline Technique preference & & & \\
First choice & $18(64.3)$ & $10(35.7)$ & n/a \\
Second choice & $10(35.7)$ & $18(64.3)$ & \\
\hline
\end{tabular}

Values are expressed as number (\%). SB: scalpel-bougie, CM: cannulato-Melker, n/a: not applicable.

omy technique within two attempts. However, the relative risk of causing posterior tracheal wall trauma after an initial failed attempt using the SB technique was three times higher than the cannula cricothyroidotomy, and twice that using the Melker Seldinger technique. This finding has not been previously reported. Perhaps this reflects the nature of the SB technique and the motor skills of the operator. An operator who is less skilled in surgical techniques may be more likely to cause inadvertent trauma when performing a more invasive technique.

According to the Fourth National Audit Project of the Royal College of Anesthetists and the Difficult Airway Society (NAP4), the CM technique is not only slower to perform when compared to the SB technique but is also associated with a high failure rate [10]. However, we did not find the higher failure rate associated with the CM technique. Our post-study questionnaire revealed that a third of anesthetists preferred to use the CM as the primary technique of emergency cricothyroidotomy. Some anesthetists prefer the CM technique because it is perceived to be less invasive and, therefore, less prone to complications, such as profuse bleeding. Others prefer it because of their familiarity with the Seldinger technique. The median duration of insertion for the cannula cricothyroidotomy was shorter than that for the SB technique (33.2 s vs. $45.2 \mathrm{~s}$ ), and this may potentially be beneficial because it allows the anesthetist to provide rescue oxygen earlier during a CICO scenario. It may be argued, however, that this potential benefit is uncertain because both the 2015 DAS Guidelines [1] and the Canadian Airway Focus Group [11] recommend that jet ventilation via an unsecured cannula should be reserved only for experienced clinicians. Further evidence on the efficacy of the CM technique in human practice is required before firm conclusions can be drawn.

Proficiency in cricothyroidotomy techniques requires regular training and not random past experiences [1]. The optimum or minimal retraining interval required for the maintenance of cricothyroidotomy skills is not known. Our department conducts an annual CICO workshop with an emphasis on cricothyroidotomy techniques. This workshop provides training and practice in the CM, SB, and Portex ${ }^{\circledR}$ cricothyroidotomy techniques using a porcine model and has the same yearly program. Since majority of participants $(82.1 \%)$ attended the departmental CICO workshop in the previous year, our data suggest that a
Table 5. Infraglottic Rescue Confidence Level

\begin{tabular}{lccc}
\hline \multicolumn{1}{c}{ Parameters } & Pre-workshop & Post-workshop & P value \\
\hline Confidence level & & & \\
Scalpel-bougie & $6.5(4.3-7.8)$ & $8(7.3-9.0)$ & $<0.001^{*}$ \\
Cannula-Melker & $5.0(1.0-7.0)$ & $8(7.0-9.0)$ & $<0.001^{*}$ \\
\hline
\end{tabular}

Values are expressed as median (IQR). Score 1 to $10,1=$ least confident, $10=$ most confident. ${ }^{*}$ Wilcoxon signed-rank test.

yearly training program is sufficient for maintenance of good performance outcomes in terms of duration of insertion and incidence of posterior tracheal wall trauma for both SB and CM techniques.

Recently, Chrisman et al. [12] conducted a similar study to evaluate the insertion time and success rate of the SB and CM compared to a new Surgicric 2 device. Similar to our results, they found that the SB technique was faster to perform and had a higher success rate when compared to the CM technique. However, we found that although the success rate at initial attempt was higher, the relative risk of causing posterior tracheal wall trauma after an initial failed attempt was higher with the SB technique. In addition, we also assessed the adequacy of yearly training.

This study has several limitations. First, we conducted the study using a porcine larynx to represent the human airway. We acknowledge that the performance of cricothyroidotomy techniques may be biased without the complexity of bleeding, obesity, edema, and difficult anatomy in a bench-top model. Furthermore, the implications of trauma due to previous failed attempts at intubation were not assessed in this model. Given the nature of the procedure, however, a human study was not feasible.

Second, we were unable to re-create the threat of an emergency situation in our study. The performance of cricothyroidotomy may differ with the addition of a stressful situation. However, we do not think that this affects the findings of our primary outcome because the CM technique requires precision during the Seldinger component and may, in fact, be even less suitable for stressful situations [1].

Third, our data suggested that a yearly retraining interval for cricothyroidotomy techniques is probably sufficient to reinforce and retain skills; however, we did not investigate the number of training cricothyroidotomy attempts required at a single time point. This has been previously addressed by Wong et al. [13]. In their mannequin study, they recommended a minimum training of five attempts to attain skill proficiency.

Fourth, when comparing the data from the 2 CICO workshops, we included participants who had not previously received the training (17.9\%). We recognize that, to assess the adequacy of yearly training, it may be better to compare data from subjects who had participated in both the 2016 and the 2017 workshops, 
and not data from subjects who had only participated in a single workshop. The purpose of our departmental CICO workshop was, however, to create a stress-free environment for all to learn and practice the various surgical airway techniques. The performances of our participants were intentionally made anonymous, and the pre- and post-workshop questionnaires were non-identifiable. Therefore, it was not possible to directly compare data from participants who had attended both workshops. Despite this, we feel that our comparison of data is still valid since majority of participants (82.1\%) attended the departmental CICO workshop in the previous year.

Fifth, our participants had different levels of experience as providers of anesthesia. We did not evaluate if this might impact their skills or evaluation of cricothyroidotomy techniques. Whilst some may be tempted to believe that having years of experience in the practice of anesthesia may confer better skills, we feel that regular training in cricothyroidotomy is more important to maintain skill proficiency because CICO events are rare.

Finally, participants were not blinded to the cricothyroidotomy techniques. However, the potential confounders, such as individual sensorimotor and cognitive skills, were minimized with a cross-over study design.

In conclusion, our study showed that the SB technique was faster to perform than the CM technique and was the preferred cricothyroidotomy method by our anesthetists. The SB technique has a high success rate, and the incidence of posterior tracheal wall trauma is comparable to that of the CM technique; however, a proficiency in the technique is required because failed SB attempts are associated with a higher likelihood of tracheal wall trauma in subsequent attempts. We advocate a yearly training program in cricothyroidotomy techniques for anesthetists to aid skill retention.

\section{Acknowledgments}

The authors would like to thank the Department of Anaesthesia and Surgical Intensive Care, Changi General Hospital, Singapore for support; Ms. Carmen Kam (MSc Statistics), a research officer at the Department of Clinical Trials and Research Unit, Changi General Hospital, Singapore for her assistance with statistical analysis; and Dr. Joanne Luo (MBBS), medical officer at the Department of Anaesthesia and Surgical Intensive Care, Changi General Hospital, Singapore for her assistance with data collection.

\section{ORCID}

See Seong Chang, https://orcid.org/0000-0002-3240-3707

Qian Jun Tong, https://orcid.org/0000-0002-7718-5272

Zhi Yuen Beh, https://orcid.org/0000-0003-0073-2546

Kelvin Howyow Quek, https://orcid.org/0000-0003-1804-6974

Bun Hui Ang, https://orcid.org/0000-0002-1164-6988

\section{References}

1. Frerk C, Mitchell VS, McNarry AF, Mendonca C, Bhagrath R, Patel A, et al. Difficult Airway Society 2015 guidelines for management of unanticipated difficult intubation in adults. Br J Anaesth 2015; 115: 827-48.

2. Cook TM, MacDougall-Davis SR. Complications and failure of airway management. Br J Anaesth 2012; 109 Suppl 1: i68-85.

3. Wong DT, Lai K, Chung FF, Ho RY. Cannot intubate-cannot ventilate and difficult intubation strategies: results of a Canadian national survey. Anesth Analg 2005; 100: 1439-46.

4. Hamaekers AE, Henderson JJ. Equipment and strategies for emergency tracheal access in the adult patient. Anaesthesia 2011; 66 Suppl 2: 65-80.

5. Baker PA, O'Sullivan EP, Kristensen MS, Lockey D. The great airway debate: is the scalpel mightier than the cannula? Br J Anaesth 2016; 117 Suppl 1: i17-9.

6. Heard A. Percutaneous emergency oxygenation strategies in the 'can't intubate, can't oxygenate' scenario [Internet]. Los Gatos: Smashworks; 2013 Nov [cited 2018 Jan]. Available from https://www.smashwords.com/books/view/377530.

7. Heard AM, Green RJ, Eakins P. The formulation and introduction of a 'can't intubate, can't ventilate' algorithm into clinical practice. Anaesthesia 2009; 64: 601-8.

8. Des Jarlais DC, Lyles C, Crepaz N. Improving the reporting quality of nonrandomized evaluations of behavioral and public health interventions: the TREND statement. Am J Public Health 2004; 94: 361-6.

9. Hubble MW, Wilfong DA, Brown LH, Hertelendy A, Benner RW. A meta-analysis of prehospital airway control techniques part II: alternative airway devices and cricothyrotomy success rates. Prehosp Emerg Care 2010; 14: 515-30.

10. Cook TM, Frerk C. Management of the 'can't intubate can't ventilate' situation and the emergency surgical airway. In: Fourth National Audit Project of the Royal College of Anaesthetists and the Difficult Airway Society. Major Complications of Airway Management in the United Kingdom. Report and Findings. Edited by Cook TM, Woodall N, Frerk C: London, Royal College of Anaesthetists. 2011, pp 105-13.

11. Law JA, Broemling N, Cooper RM, Drolet P, Duggan LV, Griesdale DE, et al. The difficult airway with recommendations for management- 
part 1-difficult tracheal intubation encountered in an unconscious/induced patient. Can J Anaesth 2013; 60: 1089-118.

12. Chrisman L, King W, Wimble K, Cartwright S, Mohammed KB, Patel B. Surgicric 2: a comparative bench study with two established emergency cricothyroidotomy techniques in a porcine model. Br J Anaesth 2016; 117: 236-42.

13. Wong DT, Prabhu AJ, Coloma M, Imasogie N, Chung FF. What is the minimum training required for successful cricothyroidotomy?: a study in mannequins. Anesthesiology 2003; 98: 349-53. 